ARTICLE

DOI: $10.1038 / s 41467-018-05615-5$

\title{
Control of transmembrane charge transfer in cytochrome $c$ oxidase by the membrane potential
}

\author{
Markus L. Björck ${ }^{1} \&$ Peter Brzezinski ${ }^{1}$
}

The respiratory chain in mitochondria is composed of membrane-bound proteins that couple electron transfer to proton translocation across the inner membrane. These charge-transfer reactions are regulated by the proton electrochemical gradient that is generated and maintained by the transmembrane charge transfer. Here, we investigate this feedback mechanism in cytochrome $c$ oxidase in intact inner mitochondrial membranes upon generation of an electrochemical potential by hydrolysis of ATP. The data indicate that a reaction step that involves proton uptake to the catalytic site and presumably proton translocation is impaired by the potential, but electron transfer is not affected. These results define the order of electron and proton-transfer reactions and suggest that the proton pump is regulated by the transmembrane electrochemical gradient through control of internal proton transfer rather than by control of electron transfer.

\footnotetext{
${ }^{1}$ Department of Biochemistry and Biophysics, The Arrhenius Laboratories for Natural Sciences, Stockholm University, SE-106 91 Stockholm, Sweden. Correspondence and requests for materials should be addressed to P.B. (email: peterb@dbb.su.se)
} 
A erobic respiration involves electron transfer from electron donors such as e.g., NADH, through a series of membrane-bound proteins, to dioxygen that is reduced to water. Part of the free energy released during this electrontransfer process is linked to proton translocation across the membrane, which results in formation of a transmembrane proton electrochemical gradient that is used for ATP production or transmembrane transport.

Transfer of electrons or protons perpendicular to the membrane surface, referred to as electrogenic events, is influenced by the electrical potential across the membrane. In other words, reactions that are involved in generating the potential are influenced by this potential. The focus of the present study is the effect of the electrochemical membrane potential on the reaction of the last component of the electron-transport chain, cytochrome $c$ oxidase $(\mathrm{Cyt} c \mathrm{O})$, with $\mathrm{O}_{2}$. In this enzyme, electrons are transferred from cytochrome $c$ to the initial electron acceptor, $\mathrm{Cu}_{\mathrm{A}}$, and then consecutively to heme $a$ and to the catalytic site composed of heme $a_{3}$ and $\mathrm{Cu}_{\mathrm{B}}$ (for review, see e.g ${ }^{1-6}$ ). Upon reduction of the catalytic site, $\mathrm{O}_{2}$ binds to heme $a_{3}$, where the molecule becomes gradually reduced to $\mathrm{H}_{2} \mathrm{O}$, which requires a total of four electrons and four protons: $4 \mathrm{e}^{-}+4 \mathrm{H}^{+}+\mathrm{O}_{2} \rightarrow 2 \mathrm{H}_{2} \mathrm{O}$. On average one proton per electron transferred to $\mathrm{O}_{2}$ at the catalytic site is pumped from the negative $(n)$ to the positive $(p)$ side of the membrane (see Fig. 1a, lower). As seen in Fig. $1 \mathrm{~b}, \mathrm{Cu}_{\mathrm{A}}$ is located near the $p$ side, while heme $a$ and the catalytic site are found within the membrane-spanning part of the protein. Hence, electrogenic reactions in $\mathrm{Cyt} c \mathrm{O}$ are electron transfer from $\mathrm{Cu}_{\mathrm{A}}$ to heme $a$, proton uptake from the $n$ side to the catalytic site as well as proton pumping ${ }^{7-9}$.

Results from earlier studies with $\mathrm{Cyt} c \mathrm{O}$ reconstituted in lipid vesicles have shown that in the presence of a transmembrane electrochemical gradient (positive on the outside) the $\mathrm{Cyt} c \mathrm{O}$ turnover is slowed typically by a factor of $5-20^{10-12}$. The effect is smaller with sub-mitochondrial particles (SMPs) where values in the range 1.4-3.5 were reported, depending on the used electron donor as well as other experimental conditions ${ }^{13,14}$.

In the present study we have investigated the effect of a transmembrane electrochemical potential on specific electron and proton-transfer events during reaction of the reduced $\mathrm{Cyt} c \mathrm{O}$ with $\mathrm{O}_{2}$ in SMPs. The potential was generated by addition of ATP, which initiates proton pumping by ATP-synthase. In this system the buildup of the electrical potential acts to lower the available free energy for proton pumping by $\mathrm{CytcO}$ (Fig. 1a, upper).

Each CytcO molecule was reduced by four electrons, one at each of the metal co-factors, $\mathrm{Cu}_{\mathrm{A}}$, heme $a$, heme $a_{3}$ and $\mathrm{Cu}_{\mathrm{B}}$. At a specific time after addition of ATP, the reaction of $\mathrm{Cyt} c \mathrm{O}$ with $\mathrm{O}_{2}$ was initiated synchronously in the entire $\mathrm{Cyt} c \mathrm{O}$ population by flash-induced dissociation of carbon monoxide from the reduced CytcO in the presence of $\mathrm{O}_{2}$. The step-wise oxidation of the CytcO was followed in time by monitoring absorbance changes associated with oxidation of hemes $a$ and $a_{3}$. The reaction sequence is described in Fig. 1b. Briefly, the dissociation of $\mathrm{CO}$ yields the reduced CytcO (called $\mathbf{R}$ ), which binds $\mathrm{O}_{2}$ to the heme $a_{3}$ iron forming a state that is called $\mathbf{A}$ with a time constant of $\sim 10 \mu$ s at 1 $\mathrm{mM} \mathrm{O}_{2}\left(\mathrm{e} . \mathrm{g}^{15}\right)$. The $\mathrm{O}-\mathrm{O}$ bond is broken and a ferryl state, called $\mathbf{P}_{\mathbf{R}},\left[\mathrm{Fe}_{\mathrm{a}_{3}}^{4+}=\mathrm{O}^{2-}\right] \quad\left[\mathrm{Cu}_{\mathrm{B}}^{2+}-\mathrm{OH}^{-}\right]$is formed at the catalytic site $e^{16-18}$ simultaneously with oxidation of heme $a^{19-21}$ and proton transfer from a nearby Tyr residue (not explicitly drawn in the Fig. 1b) with a time constant of $\sim 30 \mu$ s. Next, a proton is taken up from solution to the catalytic site leading to formation of a ferryl state called $\mathbf{F},\left[\mathrm{Fe}_{\mathrm{a}_{3}}^{4+}=\mathrm{O}^{2-}\right] \quad\left[\mathrm{Cu}_{\mathrm{B}}^{2+}-\mathrm{OH}_{2}\right]$, with a time constant of $100 \mu$ s at $\mathrm{pH} 7^{20,22,23}$. In addition, the electron at $\mathrm{Cu}_{\mathrm{A}}$

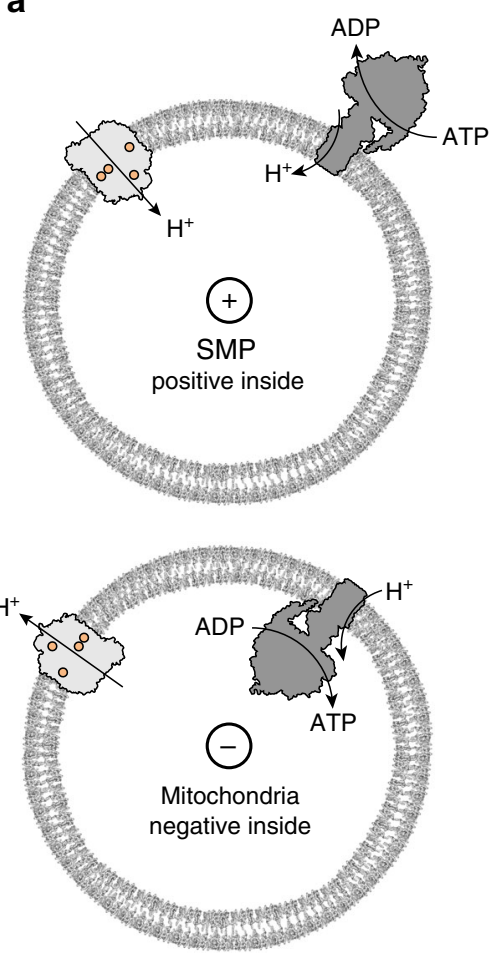

b

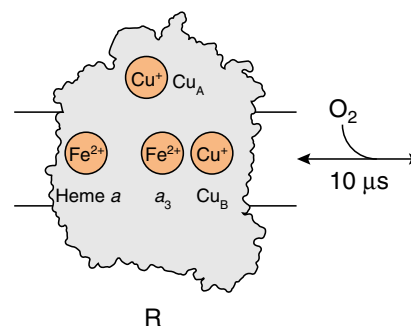

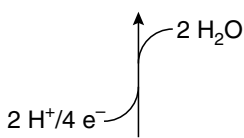

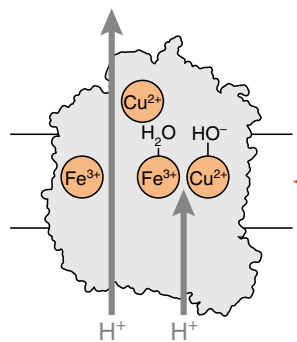

o

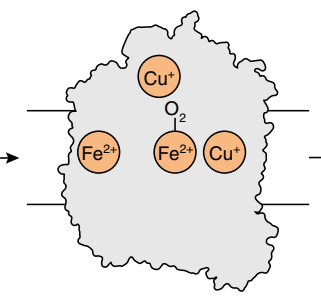

A

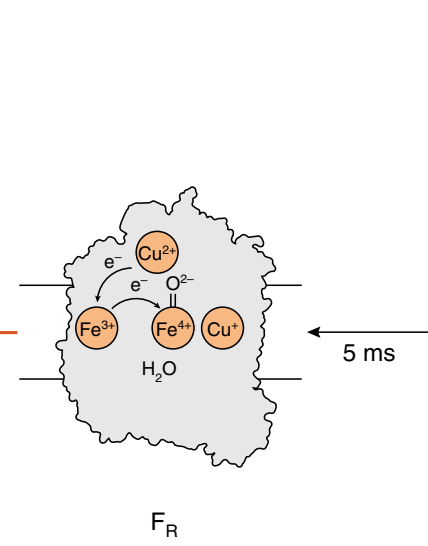

Fig. 1 Experimental system and model. a CytcO and ATP-synthase orientation in SMPs and mitochondria, respectively. $\mathbf{b}$ The reaction studied in this work. Upon initiation of the reaction by a light flash $\mathrm{CO}$ dissociates to form state $\mathbf{R}$. Then, $\mathrm{O}_{2}$ binds to the reduced $\mathrm{CytcO}$ after which the reaction proceeds as shown and described in the text. The data from this work suggest that the $\mathbf{F}_{\mathbf{R}} \rightarrow \mathbf{O}$ step is slowed/blocked by the electrochemical proton gradient (red arrow). The release of $\mathrm{H}_{2} \mathrm{O}$ is indicated in the $\mathbf{O} \rightarrow \mathbf{R}$ step, but one of the $\mathrm{H}_{2} \mathrm{O}$ molecules could be released earlier in the cycle. One of the protons taken up in this reaction is bound by a Tyr residue that is not explicitly drawn in the figure (the Tyr also donates a proton upon forming $\mathbf{P}_{\mathbf{R}}$ ) 
equilibrates with heme $a$, but in the SMPs this equilibrium is shifted toward $\mathrm{Cu}_{\mathrm{A}}$ (see below). After transfer of an electron and a proton to the catalytic site, the oxidized state, $\left[\mathrm{Fe}_{\mathrm{a}_{3}}^{3+}-\mathrm{OH}_{2}\right] \quad\left[\mathrm{Cu}_{\mathrm{B}}^{2+}-\mathrm{OH}^{-}\right], \mathbf{O}$, is formed over a millisecond time scale (in this work a 5 -ms component, see below). Typically the $\mathbf{F} \rightarrow \mathbf{O}$ reaction is observed as a single kinetic component, but results from theoretical and experimental studies indicate that it may be composed of two separable events: first electron transfer from $\mathrm{Cu}_{\mathrm{A}} /$ heme $a$ to $\mathrm{Cu}_{\mathrm{B}}$, yielding a state that is called $\mathbf{F}_{\mathbf{R}}{ }^{24-28}$ and then proton uptake to the catalytic site to yield state $\mathbf{O}$ (see Fig. 1b).

The data from the present study show that the last step of the reaction, $\mathbf{F} \rightarrow \mathbf{O}$, was influenced by membrane potential. Furthermore, the data indicate that electron transfer from heme $a$ to the catalytic site was unaffected by the membrane potential, while proton uptake and presumably pumping were slowed. As a result, state $\mathbf{F}_{\mathbf{R}}$ was formed before proton uptake and pumping. This scenario suggests that $\mathrm{Cyt} c \mathrm{O}$ turnover is regulated by altering the rate of proton transfer rather than electron transfer. Furthermore, the data define the order of electron and proton-transfer reactions during $\mathrm{Cyt} c \mathrm{O}$ turnover.

\section{Results}

Orientation of the respiratory chain in the SMPs. Results from earlier studies have shown that the orientation of the protein components of the SMPs is inverted as comparted to that of the native inner mitochondrial membrane ${ }^{29,30}$ (see Fig. 1a). To determine the orientation of the respiratory chain in our preparation we added consecutively NADH and dithionite under anaerobic conditions and compared the heme reduction levels after the additions. Because NADH does not penetrate the membrane it reduces only the redox components of the respiratory chain in SMPs with an inverted orientation. Dithionite reduces all components irrespectively of orientation. A very small further reduction was observed after addition of dithionite to the NADH-reduced membranes (Supplementary Figure 1), indicating that essentially all intact SMPs had their NADH-binding sites on the outside. Consequently, also the $\mathrm{F}_{1}$ part of the ATPsynthase was oriented to the outside of the SMPs as shown in Fig. 1a.

Formation of membrane electrochemical potential. We first studied formation of a transmembrane electrical potential $(\Delta \Psi)$ and proton concentration gradient $(\Delta \mathrm{pH})$ in the SMPs upon addition of ATP. Figure 2a shows changes in absorbance at 623 $\mathrm{nm}, \Delta A^{623}$, as a function of time after addition of ATP to SMPs in the presence of the membrane potential-sensitive dye oxonol $\mathrm{VI}^{31}$. As seen in the figure, the absorbance increased, which indicates that the potential increased. The initial increase was transient after which it decreased over a time scale of $\sim 200 \mathrm{~s}$ to reach a slow decay slope. The inset shows a similar experiment, but done in a stopped-flow apparatus with a higher time resolution. In the inset it is seen that the maximum of the transient potential change was observed after $\sim 3 \mathrm{~s}$. Upon addition of nigericin, which equilibrates the potassium and proton concentration gradients to convert the $\Delta \mathrm{pH}$ component into a $\Delta \Psi$ (in the current experiment where protons are continuously pumped by the ATP synthase), the absorbance increased further (Fig. 2a). No changes in absorbance were observed in the presence of valinomycin, which abolishes $\Delta \Psi$ but not $\Delta \mathrm{pH}$. A calibration curve done with liposomes of the same size as the SMPs is shown in Supplementary Figure 2. Based on these data we estimated the membrane electrical potential established across the SMPs to be $\sim 100 \mathrm{mV}$ and the $\Delta \mathrm{pH}$ at the time of nigericin addition to be $\sim 0.4 \mathrm{pH}$ units (see Fig. 2a).

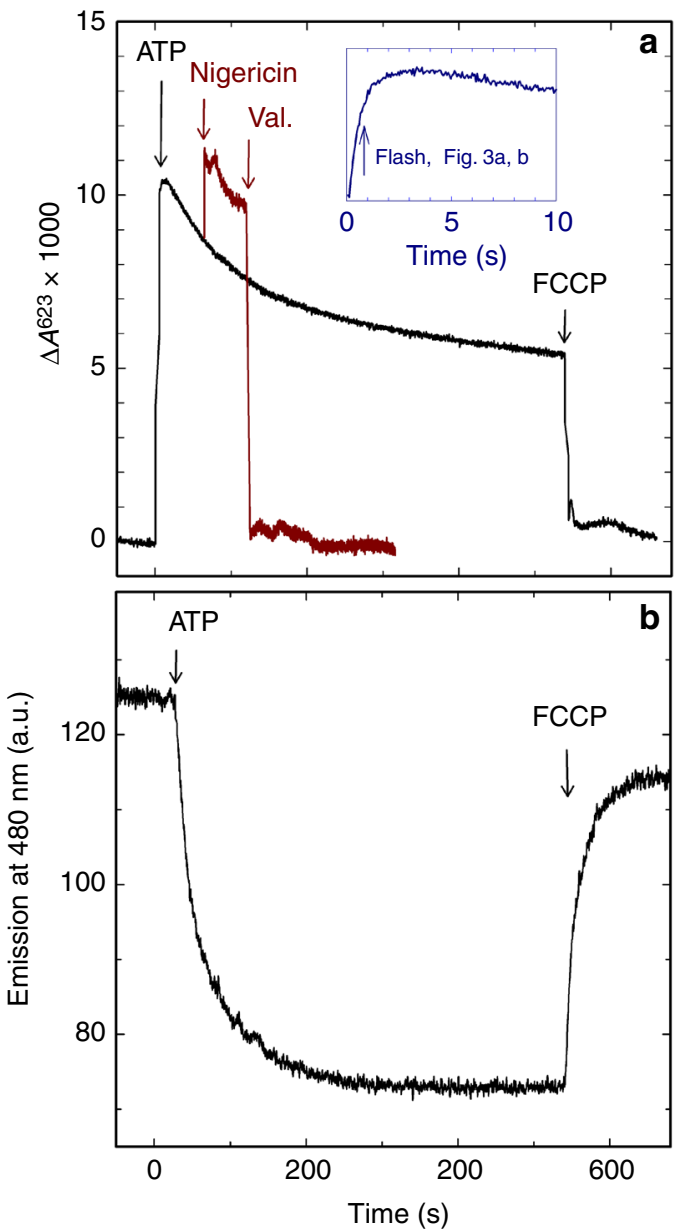

Fig. 2 Generation of an ATP-induced transmembrane electrochemical potential in SMPs. a The membrane electrical potential $(\Delta \Psi)$ was monitored using the dye oxonol VI. The absorbance changes at $623 \mathrm{~nm}$ were measured as a function of time. ATP was added to the SMPs solution, containing oxonol $\mathrm{VI}$, at $t=0$ (black trace). The signal increased, consistent with proton pumping into the SMPs. Addition of nigericin after $\sim 60 \mathrm{~s}$ (red trace) resulted in an increase in absorbance as the proton gradient $(\Delta \mathrm{pH})$ was converted into an electrical potential. In the presence of valinomycin, (val., added after $\sim 120 \mathrm{~s}$ ) the absorbance decreased to the same level as that before addition of ATP (red trace). Addition of FCCP (at $~ 540 \mathrm{~s}$ ) removes both $\Delta \Psi$ and $\Delta \mathrm{pH}$ (black trace). Mixing artifacts caused by the additions were removed for clarity. The absorbance changes were also resolved on a shorter time scale using a stopped-flow device (see inset in a). The arrow indicates the time at which the reaction was started in the experiments shown in Fig. 3a, b. Experimental conditions: $0.1 \mathrm{mg}$ SMPs in 1 $\mathrm{ml}$ buffer, $2 \mu \mathrm{M}$ oxonol $\mathrm{VI}, 1 \mu \mathrm{M}$ valinomycin. The final ATP concentration was $160 \mu \mathrm{M}$. In the stopped-flow experiment it was $1 \mathrm{mM}$. b Proton pumping to the interior of the SMPs (initiated by addition of ATP at $t=0$ ) was monitored using the fluorescent dye ACMA. As the inside of the SMPs became acidified the emission of the fluorescent dye was quenched. Experimental conditions: $0.1 \mathrm{mg}$ SMPs in $1 \mathrm{ml}$ buffer, $200 \mathrm{nM}$ ACMA, the final ATP concentration was $160 \mu \mathrm{M}$. The fluorophore was excited at 410 $\mathrm{nm}$ and the emission was recorded at $480 \mathrm{~nm}$. (a.u.) is arbitrary units

Changes in the transmembrane $\mathrm{pH}$ gradient were monitored by measuring fluorescence changes of the dye 9-amino-6-chloro2-methoxyacridine (ACMA) (Fig. 2b). The fluorescence decreased upon proton pumping into the SMPs ${ }^{32}$ over a time scale of $\sim 200$ s, i.e., the same time scale as the initial fractional decay in $\Delta \Psi$. Addition of the proton ionophore FCCP eliminated the fluorescence signal. 
The initial transient increase in membrane potential was much faster $(\sim 3 \mathrm{~s})$ than formation of the proton concentration gradient $(\sim 100 \mathrm{~s})$, which is consistent with results from earlier studies ${ }^{31}$. This difference in time scales is observed because formation of $\Delta \Psi$ requires transfer of only a few charges across the membrane (charging of a spherical lipid-membrane capacitor), while formation of $\Delta \mathrm{pH}$ requires a large number of turnovers by the ATP synthase. The decay in the membrane potential, starting at $\sim 5 \mathrm{~s}$ after addition of ATP, is presumably caused by equilibration of proton pumping and ion leaks being established across the membrane.

We also measured the $\mathrm{O}_{2}$-reduction rate upon addition of succinate to the SMPs in the presence and absence of the proton ionophore FCCP. The ratio of these rates is defined as the respiratory control ratio (referred to as RCR). We obtained an RCR value of $1.30 \pm 0.05$ (SD of 10 measurements).

Reaction with $\mathrm{O}_{2}$. We reduced the SMPs with ascorbate and incubated the sample under an atmosphere of $\mathrm{CO}$, which resulted in formation of the reduced $\mathrm{Cyt} c \mathrm{O}-\mathrm{CO}$ complex. This sample solution was mixed 1:1 with an $\mathrm{O}_{2}$-saturated $\left(\sim 1.2 \mathrm{mM} \mathrm{O}_{2}\right)$ solution containing ATP in a stopped-flow apparatus. After a time delay of $\sim 0.8 \mathrm{~s}$ the $\mathrm{CO}$ ligand was dissociated by means of a laser flash, which allowed $\mathrm{O}_{2}$ to bind to initiate the reaction of the reduced CytcO with $\mathrm{O}_{2}$. During the time window of mixing and initiation of the reaction, an electrical potential was established across the membrane, but the proton concentration gradient was expected to be small after $\sim 0.8 \mathrm{~s}$ (c.f. Fig. $2 \mathrm{a}, \mathrm{b}$ ).

The reaction was monitored at two wavelengths, $445 \mathrm{~nm}$ and $605 \mathrm{~nm}$. At $445 \mathrm{~nm}$ hemes $a$ and $a_{3}$ contribute by $\sim 40 \%$ and $\sim 60 \%$, respectively, to the total reduced-minus-oxidized difference spectrum ${ }^{33}$. At $605 \mathrm{~nm}$ the main contribution is from redox changes at heme $a$, which contributes by $\sim 80 \%$ of the total change 33,34 .

At $445 \mathrm{~nm}$ the absorbance increased at the time of the laser flash ( $t=0$, Fig. 3a), which is associated with dissociation of the $\mathrm{CO}$ ligand. The absorbance level immediately after illumination corresponds to that of the reduced CytcO (state $\mathbf{R}$, see scheme in Fig. 1b). The decrease in absorbance at both $445 \mathrm{~nm}$ and $605 \mathrm{~nm}$ (Fig. 3b) is associated with $\mathrm{O}_{2}$ binding to heme $a_{3}$ forming state $\mathbf{A}$ ( $\tau \cong 20 \mu$ s at $0.5 \mathrm{mM} \mathrm{O}_{2}$ ) and formation of the peroxy state, $\mathbf{P}_{\mathbf{R}}$, which is associated with electron transfer from heme $a$ to the catalytic site $(\tau \cong 30 \mu$ s).

In the next step a proton is transferred to the catalytic site to form the ferryl, $\mathbf{F}$ state with a time constant of $\sim 100 \mu$ s at $\mathrm{pH} 7$. The $\mathbf{P}_{\mathbf{R}} \rightarrow \mathbf{F}$ reaction is associated with pumping of one proton across the membrane and occurs over the same time scale as a shift in the electron equilibrium from $\mathrm{Cu}_{\mathrm{A}}$ toward heme $a$. Results from earlier studies have shown that this electron transfer yields an absorbance increase or plateau in the time range approximately 200-500 $\mu$ s at $445 \mathrm{~nm}$, depending on the fraction reduced heme $a^{20}$. With the bovine CytcO in detergent solution a relatively large increase in absorbance was $\operatorname{seen}^{20}$, but in the SMPs we observed only a plateau, indicating a small fractional electron transfer from $\mathrm{Cu}_{\mathrm{A}}$ to heme $a$, also without a membrane potential (see Fig. 3a). In the last step of the reaction the fourth electron is transferred from heme $a$ to the catalytic site, which yields the oxidized Cyt $c \mathrm{O}$ (state $\mathbf{O}$ ). The $\mathbf{F} \rightarrow \mathbf{O}$ transition $(\tau \cong 5$ $\mathrm{ms}$ ) is linked to proton uptake to the catalytic site and pumping across the membrane, which are both electrogenic. The time constant of the reaction is typically $\sim 1 \mathrm{~ms}$ in detergent solution ${ }^{35}$, but in the SMPs it was slowed to $\sim 5 \mathrm{~ms}$ (Fig. $3 \mathrm{AB}$ ), as observed previously for membrane-bound $\mathrm{CytcO}^{36}$.

The amplitude of the absorbance change at $445 \mathrm{~nm}$ decreased by $14 \pm 1 \%$ (SD, 3 measurements) in the presence of a membrane
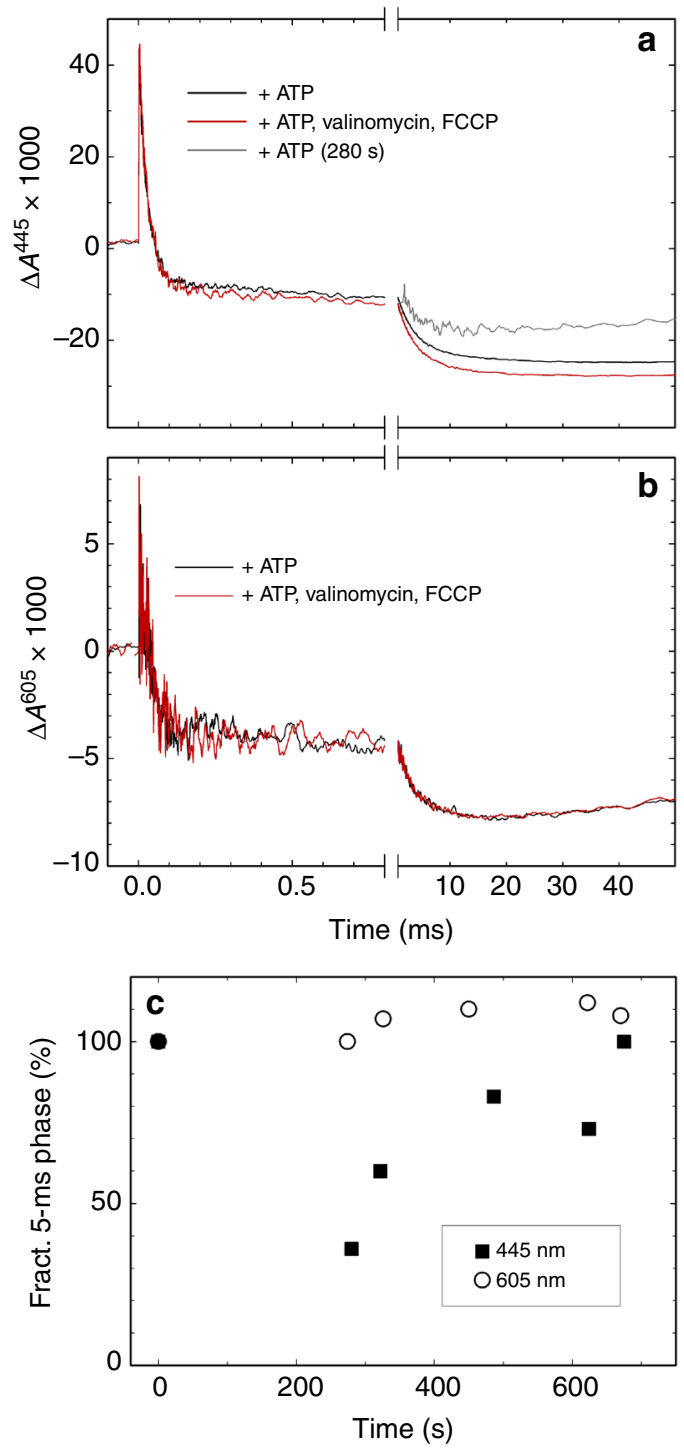

Fig. 3 Absorbance changes associated with the reaction of $\mathrm{CytcO}$ with $\mathrm{O}_{2}$ in sub-mitochondrial particles. The reduced $\mathrm{CytcO}$ was mixed with an $\mathrm{O}_{2-}$ saturated solution containing ATP. After $0.8 \mathrm{~s}$ the reaction of the CytcO with $\mathrm{O}_{2}$ was initiated by a laser flash (at $t=0$ in the graph). It was monitored at $445 \mathrm{~nm}$ (a), which reflects the redox states of hemes $a$ and $a_{3}$, and at $605 \mathrm{~nm}$ (b), which reflects the redox state mainly of heme $a$. Experimental conditions after mixing: $250 \mathrm{mM}$ sucrose, $50 \mathrm{mM} \mathrm{KCl}, 10 \mathrm{mM}$ phosphate buffer at $\mathrm{pH} 7.4,5 \mathrm{mM} \mathrm{MgCl} 2,0.1 \mathrm{mM}$ EDTA, $25 \mathrm{mM}$ ATP, 1.9 $\mathrm{mg} \mathrm{ml}^{-1}$ SMPs with or without $10 \mu \mathrm{M}$ valinomycin and $200 \mathrm{nM} \mathrm{FCCP.} \mathrm{The}$ mixing ratio was 1:1. The grey graph is the 280-s time point from $\mathbf{c}$. Typically, 10 or 20 traces were averaged at $445 \mathrm{~nm}$ and $605 \mathrm{~nm}$, respectively. In $\mathbf{b}$ a laser artifact has been truncated. $\mathbf{c}$ Amplitude of the 5ms component as a function of time monitored at $445 \mathrm{~nm}$ (filled symbols) and $605 \mathrm{~nm}$ (open symbols). ATP was added to the anaerobic SMP solution before the sample was transferred to the stopped-flow apparatus. The first time point in this graph was obtained before addition of ATP (set to $100 \%$ ). The errors, estimated from the noise level of the traces, were typically smaller than the marks. Experimental conditions before mixing: $250 \mathrm{mM}$ sucrose, $50 \mathrm{mM} \mathrm{NaCl}, 20 \mathrm{mM}$ Hepes pH 7.4, $5 \mathrm{mM} \mathrm{MgCl}, 1 \mathrm{mM}$ ATP and $5.5 \mathrm{mg} \mathrm{ml}^{-1} \mathrm{SMP}$. The $\mathrm{SMP}: \mathrm{O}_{2}$ solution mixing ratio was 1:5. The time delay between mixing and laser flash was $0.2 \mathrm{~s}$ 
potential (Fig. 3a). At $605 \mathrm{~nm}$ the difference in the amplitudes without and with a membrane potential for the 5-ms component was $<2 \%$ (3 measurements) (Fig. 3b). A comparison of the absorbance changes at these two wavelengths indicates that with a membrane potential, electrons remained at the catalytic site at the end of the reaction (after $\sim 10 \mathrm{~ms}$ ), while heme $a$ was essentially fully oxidized independently of the membrane potential.

The experiments described above were carried out in a stopped-flow apparatus where ATP and $\mathrm{O}_{2}$ were added at the same time. Therefore, the delay time between addition of $\mathrm{O}_{2}$ (and thus ATP) and flash-induced initiation of the reaction must be kept short enough to prevent $\mathrm{O}_{2}$ from reacting before flash photolysis of the CO ligand. To increase the time between ATP addition and start of the reaction, we also carried out an experiment in which ATP was added to the SMP-containing cuvette in the absence of $\mathrm{O}_{2}$. The sample was then introduced into the stopped-flow apparatus and mixed with $\mathrm{O}_{2}$ as described above. Because it took $\sim 5$ minutes for the anaerobic transfer of the sample to reach the cuvette, the first time point after addition of ATP was measured after $280 \mathrm{~s}$ (Fig. 3c). As seen in Fig. 3a (grey trace), at this time the amplitude of the 5-ms component in the absorbance change had decreased to $\sim 50 \%$ of its maximum value (100\% was set as the amplitude before ATP addition). This drop in amplitude is larger than that observed after $0.8 \mathrm{~s}$ incubation (see above) (Fig. 3a) presumably because at $280 \mathrm{~s}$ the $\mathrm{pH}$ gradient was fully developed (c.f. Figure 2). At times $>280$ $\mathrm{s}$ the amplitude increased again to reach $\sim 100 \%$ at $\sim 600 \mathrm{~s}$, presumably when $\Delta \Psi$ decreased (see slow decrease in absorbance at times $>280 \mathrm{~s}$, Fig. 2a), while $\Delta \mathrm{pH}$ remained at a constant level (see Fig. 2b). As seen in Fig. 3c, the amplitude of the 5-ms component at $605 \mathrm{~nm}$ remained at an approximately constant level during the same time period after addition of ATP.

\section{Discussion}

We have investigated the effect of membrane potential on the reaction of the four-electron reduced CytcO with $\mathrm{O}_{2}$ in SMPs. No effect of membrane potential on $\mathbf{P}_{\mathbf{R}}$ formation was observed ( $\tau \cong 30 \mu \mathrm{s}$ ). This reaction step involves electron transfer from heme $a$ to the catalytic site, which occurs parallel to the membrane surface. Hence, this electron transfer is expected to be insensitive to the external potential. The following two reaction steps are linked to proton uptake and pumping, and electron transfer perpendicular to the membrane plane; $\mathbf{P}_{\mathbf{R}} \rightarrow \mathbf{F}$ and $\mathbf{F} \rightarrow \mathbf{O}$ (see Fig. 1b). Results from earlier studies showed that both these steps are electrogenic ${ }^{8,37}$, which suggests that both reactions could, in principle, be sensitive to an external potential.

We discuss the last step of $\mathrm{O}_{2}$ reduction to $\mathrm{H}_{2} \mathrm{O}$, the $\mathbf{F} \rightarrow \mathbf{O}$ reaction, first. It involves simultaneous electron transfer from the $\mathrm{Cu}_{\mathrm{A}}$-heme $a$ equilibrium to the catalytic site, proton uptake to the catalytic site and proton pumping. In other words, the $\mathbf{F} \rightarrow \mathbf{O}$ reaction is representative of each step in $\mathrm{Cyt} c \mathrm{O}$ turnover where electrons are delivered one-by-one from cytochrome $c$, via CytcO, to $\mathrm{O}_{2}$. The electron and proton-transfer reactions in $\mathbf{F} \rightarrow \mathbf{O}$ occur over the same time scale and are presumably rate-limited by the release of the pumped proton ${ }^{38-40}$.

At $605 \mathrm{~nm}$ the absorbance changes with and without electrochemical potential were nearly identical over a time scale of $\sim 10$ ms (Fig. $3 \mathrm{~b}$ and $\mathrm{c}$ ), which shows that the extent of heme $a$ oxidation during the time of the $\mathbf{F} \rightarrow \mathbf{O}$ reaction was independent of the membrane potential. However, the amplitude of the absorbance change at $445 \mathrm{~nm}$ decreased in the presence of membrane potential (Fig. 3a), which qualitatively indicates less oxidation of the catalytic site. Collectively, these observations indicate that all four electrons are transferred to $\mathrm{O}_{2}$ at the catalytic site over a time scale of $5 \mathrm{~ms}$, but that this electron transfer does not lead to complete reduction of the bound $\mathrm{O}_{2}$ to $\mathrm{H}_{2} \mathrm{O}$, i.e., that proton uptake to form $\mathrm{H}_{2} \mathrm{O}$ is impaired.

As discussed in the Introduction section, the reaction steps that are part of the $\mathbf{F} \rightarrow \mathbf{O}$ reaction have been considered in detailed theoretical studies 24,25 . It was suggested that over the time scale of $\mathbf{F} \rightarrow \mathbf{O}$ reaction, the electron is initially transferred to $\mathrm{Cu}_{\mathrm{B}}{ }^{2+}$ yielding state $\mathbf{F}_{\mathbf{R}}{ }^{26}$ (see Fig. $1 \mathrm{~b}$ ), which was also indicated from experiments ${ }^{27,28}$. As already noted above, electron transfer from heme $a$ to $\mathrm{Cu}_{\mathrm{B}}$ occurs parallel to the membrane surface, and it is therefore expected to be insensitive to the membrane potential. Reduction of $\mathrm{Cu}_{\mathrm{B}}$ is followed in time by proton uptake to complete $\mathrm{O}_{2}$ reduction to $\mathrm{H}_{2} \mathrm{O}$ and proton pumping across the membrane. In principle, the protonpumping event could occur over the time scale of either the $\mathbf{F}$ $\rightarrow \mathbf{F}_{\mathbf{R}}$ or the $\mathbf{F}_{\mathbf{R}} \rightarrow \mathbf{O}$ reaction, but it is more likely to coincide with the latter in analogy with proton pumping during the $\mathbf{P}_{\mathbf{R}} \rightarrow$ F reaction, i.e. the previous step ${ }^{36,41}$ (see Fig. 1b). Furthermore, we note that upon one-electron reduction of $\mathrm{CytcO}$ in the $\mathbf{F}$ state, the $\mathbf{F} \rightarrow \mathbf{O}$ reaction was found to be biphasic with a second, slower component accounting for $3 / 4$ of the total electrogenicity ${ }^{28}$. These two components would be associated with electron $\left(\mathbf{F} \rightarrow \mathbf{F}_{\mathbf{R}}\right)$ and proton $\left(\mathbf{F}_{\mathbf{R}} \rightarrow \mathbf{O}\right)$ transfer (as well as pumping), respectively. Because proton uptake and pumping have trajectory components that are perpendicular to the membrane surface, the driving force $(-\Delta G)$ of these reactions is expected to be smaller with than without a membrane potential, i.e. the $\mathbf{F}_{\mathbf{R}} \rightarrow \mathbf{O}$ reaction would be impaired.

As pointed out above, we assume that in the absence of membrane potential the two reaction steps, $\mathbf{F} \rightarrow \mathbf{F}_{\mathbf{R}}$ and $\mathbf{F}_{\mathbf{R}} \rightarrow \mathbf{O}$, are inseparable in time, presumably because decay of state $\mathbf{F}_{\mathbf{R}}$ to $\mathbf{O}$ is faster than its formation. This assumption also explains why the time constant of the $\mathbf{F} \rightarrow \mathbf{F}_{\mathbf{R}}$ reaction with a transmembrane potential is the same as that of the $\mathbf{F} \rightarrow \mathbf{O}$ reaction without a membrane potential; the time constant for electron transfer from heme $a$ to $\mathrm{Cu}_{\mathrm{B}}$ to form $\mathbf{F}_{\mathbf{R}}$ is not affected by the membrane potential, while the next reaction step, $\mathbf{F}_{\mathbf{R}} \rightarrow \mathbf{O}$, is slowed/impaired such that the state formed over the time scale of measurement is $\mathbf{F}_{\mathbf{R}}$. Furthermore, the $\sim 14 \%$ decrease of the absorbance amplitude at $445 \mathrm{~nm}$ upon formation of a membrane potential would reflect the contribution of $\mathbf{F}_{\mathbf{R}}$, relative to that of $\mathbf{O}$.

Assuming the scenario outlined above, the amplitude of the 5ms component in the presence of membrane potential would reflect electron transfer from heme $a$ to $\mathrm{Cu}_{\mathrm{B}}$, i.e., mainly oxidation of heme $a$, for which the absorption coefficient at $445 \mathrm{~nm}$ is $\sim 60 \mathrm{mM}^{-1} \mathrm{~cm}^{-133,34}$. The absorption coefficient for state $\mathbf{F}$ (relative to the oxidized CytcO) is $\sim 10 \mathrm{mM}^{-1} \mathrm{~cm}^{-17}$. Thus, if $\mathbf{F}$ with an oxidized heme $a$ was the final state after $5 \mathrm{~ms}$, we would expect a decrease of the 5 -ms component amplitude by $\sim 15 \%$. This number is approximately equal to the observed $14 \%$ change in amplitude. However, the absorption coefficient for state $\mathbf{F}_{\mathbf{R}}$ may differ from that of state $\mathbf{F}$ because of the additional electron at $\mathrm{Cu}_{\mathrm{B}}$ in the former, which may explain why we observed an even bigger decrease in the 445-nm amplitude for the data shown in Fig. 3c. The contribution of state $\mathbf{F}$ at $605 \mathrm{~nm}$ is $\sim 5 \%$ of that of heme $a^{7,42}$, which explains why the absorbance levels were the same after $5 \mathrm{~ms}$ at this wavelength.

The conclusion from the discussion above is that in the presence of an electrochemical potential the $\mathrm{Cyt} c \mathrm{O}$ state reached after the 5-ms component is $\mathbf{F}_{\mathbf{R}}$. In other words, the decrease in the amplitude of this component is a reflection of a considerable decrease in the rate of the next reaction step, i.e., $\mathbf{F}_{\mathbf{R}} \rightarrow \mathbf{O}$. It is difficult to estimate the decay time constant of this state in the SMPs because after the CytcO is oxidized it becomes slowly rereduced by ascorbate that must be present in the experiment for preparation of the reduced form of the enzyme. Nevertheless, as 
seen in Fig. 3a the absorbance level was essentially unaltered for about $50 \mathrm{~ms}$, which defines the lower limit for the life time of $\mathbf{F}_{\mathbf{R}}$.

We now turn to the $\mathbf{P}_{\mathbf{R}} \rightarrow \mathbf{F}$ reaction. The absorbance changes associated with this reaction are very small, also at the peak wavelength of $\sim 580 \mathrm{~nm}^{7}$ and could not be resolved here with the scattering SMPs. As discussed above, the $\mathbf{P}_{\mathbf{R}} \rightarrow \mathbf{F}$ reaction, and proton translocation that is linked to the reaction ${ }^{36,40,43}$ occur over the same time scale as a shift in the equilibrium between $\mathrm{Cu}_{\mathrm{A}}$ and heme $a$, leading to a fractional reduction of heme $a^{44}$. This shift yields absorbance changes, for example, at $445 \mathrm{~nm}$ and at $605 \mathrm{~nm}$, associated with reduction of heme $a$. However, the extent of heme $a$ reduction varies between CytcOs from different species $^{20}$ and depending on experimental conditions (detergent, lipids, $\mathrm{pH}$ etc.) $\left(\mathrm{e} . \mathrm{g}^{22}\right)$. For the SMPs studied here the extent of the $\mathrm{Cu}_{\mathrm{A}}$-heme $a$ electron transfer was small during the $\mathbf{P}_{\mathbf{R}} \rightarrow \mathbf{F}$ reaction (Fig. 3, also indicated in Fig. 1b). We did not observe any differences in the absorbance changes with or without a membrane potential over the time scale of the $\mathbf{P}_{\mathbf{R}} \rightarrow \mathbf{F}$ reaction, presumably because the membrane potential established by ATP hydrolysis acts to shift the $\mathrm{Cu}_{\mathrm{A}}$-heme $a$ electron equilibrium even further toward $\mathrm{Cu}_{\mathrm{A}}{ }^{11}$. It should be noted that the proton uptake and pumping during $\mathbf{P}_{\mathbf{R}} \rightarrow \mathbf{F}$ are independent of the electron transfer from $\mathrm{Cu}_{\mathrm{A}}$ to heme $a^{44}$. Because heme $a$ was oxidized over a time scale of $\sim 5 \mathrm{~ms}$ (see Fig. $3 \mathrm{~b}$ ), the $\mathbf{P}_{\mathbf{R}} \rightarrow \mathbf{F}$ reaction must occur with a time constant $\leq 5 \mathrm{~ms}$, i.e. it is not rate limiting for the overall oxidation of $\mathrm{Cyt} c \mathrm{O}$.

Data from earlier studies of the $\mathbf{P}_{\mathbf{R}} \rightarrow \mathbf{F}$ reaction in the $R$. sphaeroides Cytc $\mathrm{O}$ in $\mathrm{H}_{2} \mathrm{O}$ and $\mathrm{D}_{2} \mathrm{O}$, respectively, showed that the ratio of the proton-release rates was $\sim 7$ (defined as kinetic isotope effect, KIE) ${ }^{38}$. In contrast, the KIE of the $\mathbf{P}_{\mathbf{R}} \rightarrow \mathbf{F}$ chemical reaction and the associated proton-uptake reactions was $2-3^{45}$. These differences in the KIEs yielded a delay of the proton release, relative to proton uptake in $\mathrm{D}_{2} \mathrm{O}$. This means that even if the release of the pumped proton would be slowed or impaired by the membrane potential, the $\mathbf{F}$ state could be formed at a rate that is independent of the membrane potential.

We measured a RCR of $\sim 1.3$, which is at the lower end of the range of previously reported values ${ }^{13,14}$. Nevertheless, the value is significantly larger than unity and both the electrical component and $\mathrm{pH}$ gradient remained stable during the time of the measurements (c.f. Figure 2). Furthermore, we note that in the earlier studies of the RCR oligomycin was used to block proton leaks via the ATP synthase. Because in the current study we used the ATP synthase to maintain a transmembrane electrochemical potential, oligomycin had to be excluded.

The mechanistic scenario suggested from this work defines the order of electron and proton transfer during $\mathrm{O}_{2}$ reduction at the CytcO catalytic site. Electron transfer from heme $a, \mathbf{F} \rightarrow \mathbf{F}_{\mathbf{R}}$, occurs first and is insensitive to the membrane potential. This electron transfer may be linked to proton uptake from the $n$ side of the membrane to a proton-loading site (PLS) ${ }^{43,46,47}$, but not to the catalytic site. Uptake of a proton to the catalytic site, $\mathbf{F}_{\mathbf{R}} \rightarrow \mathbf{O}$, and release of the pumped proton is significantly slowed by the membrane potential. Alternatively, only the electron transfer from heme $a$ to the catalytic site $\left(\mathbf{F} \rightarrow \mathbf{F}_{\mathbf{R}}\right)$, parallel to the membrane surface, is insensitive to the membrane potential, in agreement with a model proposed earlier ${ }^{36,41}$. Both scenarios would imply that electron transfer to the catalytic site can occur without accompanying proton uptake, that release of the pumped proton from PLS is mechanistically linked to the proton uptake to the catalytic site and that the latter reaction is influenced by the electrochemical proton gradient. We note that the electrochemical potential created in the SMPs was somewhat lower than that found in the native system, i.e., the effects were observed at potentials lower than that found in vivo.
The data from these studies suggest a more general mechanism by which the cell may regulate the rate of respiration. It has been shown that CytcO is a key site for regulation of the OXPHOS ${ }^{48}$. Even though there are several modes of regulation of the enzyme (see e..$^{1,48-51}$ ) modulation of the turnover rate by changes in the proton electrochemical potential is a general principle. The data from the present study indicate that the $\mathrm{CytcO}$ turnover is regulated by slowing proton uptake and presumably pumping in the $\mathbf{F}_{\mathbf{R}} \rightarrow \mathbf{O}$ reaction. Because during CytcO turnover, formation of $\mathbf{O}$ is followed in time by re-reduction, also electron transfer would be slowed by the membrane potential. This mode of regulation is consistent with the three-dimensional arrangement of the proton pathways and metal cofactors in $\mathrm{Cyt}_{c} \mathrm{O}^{52-55}$. Proton uptake is perpendicular to the membrane surface and takes place across $2 / 3$ of the transmembrane distance, across a hydrophobic region of the $\mathrm{Cyt} c \mathrm{O}$ where the dielectric constant is expected to be small. Proton pumping spans across the entire distance of the membrane dielectric. Hence, the driving force of the protontransfer reactions is expected to be sensitive to the transmembrane potential. Electron transfer from $\mathrm{Cu}_{\mathrm{A}}$ to heme $a$, on the other hand, takes place across only $1 / 3$ of the transmembrane distance, through a region of the protein that is likely to have a relatively large dielectric constant because of the large amount of water molecules ${ }^{52-55}$. In conclusion, the data suggest that the activity of CytcO is regulated by the external electrochemical potential as a result of altering the driving force for proton transfer in the very last step of $\mathrm{O}_{2}$ reduction to $\mathrm{H}_{2} \mathrm{O}$.

\section{Methods}

Reagents. All chemicals used in this study were of the purest grade available and purchased from Sigma Aldrich.

Preparation of bovine heart mitochondria. Bovine heart mitochondria were prepared as described in ${ }^{56}$. Procedure three was used with the following modifications: $600 \mathrm{ml}$ sucrose buffer was used in the blender step and $45 \mathrm{~s}$ pulses were used instead of $15 \mathrm{~s}$, the homogenate was centrifuged at $1600 \mathrm{x}$ for $15 \mathrm{~min}$ in the first centrifugation step, and at $10,000 \mathrm{x} g$ for $30 \mathrm{~min}$ in the following steps. The mitochondria were separated into light and heavy fractions as described ${ }^{56}$. Briefly, the sample containing mitochondria was centrifuged at $10,900 \mathrm{x} g$. The pellet consisted of two parts, one light, slightly less colored and one darker more firm pellet. The lighter fraction (broken mitochondrial fragments) was removed by decantation. The procedure was then repeated until only the heavy fraction of mitochondria was present after centrifugation.

Preparation of SMPs. A sample containing the heavy fraction bovine heart mitochondria was diluted to $\sim 3.3 \mathrm{mg} \mathrm{ml}^{-1}$ in a buffer containing $250 \mathrm{mM}$ sucrose, $50 \mathrm{mM} \mathrm{KCl}, 10 \mathrm{mM}$ phosphate-buffer at $\mathrm{pH} 7.4,5 \mathrm{mM} \mathrm{MgCl}_{2}$ and $0.1 \mathrm{mM}$ EDTA, and the solution was homogenized in a glass potter. The mitochondrial suspension was then sonicated for $1 \mathrm{~min}$ at maximum output using a Vibra cell sonicator (Sonics \& Materials Inc.) while cooled using a mixture of ice and water. The sonicated solution was diluted 1:1 in sucrose buffer and then centrifuged at 14,000x $g$ for 10 min to remove debris and intact mitochondria. The pellet was discarded and the supernatant was centrifuged at $10,5000 \mathrm{x} g$ for $1 \mathrm{~h}$. The supernatant was discarded and the pellet was dissolved in the same buffer as previously to a concentration in the range $10-20 \mathrm{mg} \mathrm{ml}^{-1}$. The SMPs were flash-frozen in liquid nitrogen and stored at $-80^{\circ} \mathrm{C}$ until use.

Flow-flash measurements. The SMPs were dissolved in the sucrose buffer described above to a concentration of $\sim 3.8 \mathrm{mg} \mathrm{ml}^{-1}$. The sample was loaded into a Thunberg cuvette and air was exchanged for nitrogen. The sample was then reduced with $4 \mathrm{mM}$ ascorbate and $1 \mu \mathrm{M}$ PMS. After the sample was fully reduced, nitrogen was exchanged for $\mathrm{CO}$. The absorbance changes associated with reduction and binding of $\mathrm{CO}$ to $\mathrm{Cyt} c \mathrm{O}$ were monitored using a Cary 4000 spectrophotometer (Agilent).

The flow-flash measurements were performed essentially as described previously ${ }^{57,58}$ using a laser flash-photolysis system combined with a stopped-flow apparatus (Applied Photophysics). The mixing ratio of sample and saturated oxygen buffer was $1: 1$ and the cuvette path length was $1.00 \mathrm{~cm}$. The delay time between mixing and laser flash was $0.8 \mathrm{~s}$. The oxygen-containing buffer consisted of the sucrose buffer used to dissolve SMPs and it also contained either only ATP or ATP, $200 \mathrm{nM}$ FCCP and $10 \mu \mathrm{M}$ valinomycin. The ATP concentration after mixing was $25 \mathrm{mM}$. 
Measurement of membrane potential and proton pumping. The membrane potential generated upon addition of ATP to SMPs was monitored using the potential-sensitive dye Oxonol VI $\left(\mathrm{see}^{59}\right)$. SMPs were dissolved in sucrose buffer (see above) at a concentration of $0.1 \mathrm{mg} \mathrm{ml}^{-1}$. Changes in absorbance were monitored at $623 \mathrm{~nm}$ using a Cary 100 spectrophotometer (Agilent). The dye oxonol VI was then added to a final concentration of $2 \mu \mathrm{M}$. Nigericin was added (1 $\mu \mathrm{M})$ to convert the $\mathrm{pH}$ gradient into potential under the conditions of these measurements. As a control, valinomycin was added and finally FCCP ( $1 \mu \mathrm{M}$ final concentration of each) to dissipate the membrane potential and proton gradient. The final volume after the additions was $1 \mathrm{ml}$ and final ATP concentration was 160 $\mu \mathrm{M}$.

A stopped-flow apparatus, equipped with a diode-array detector (Applied Photophysics), was used to increase the time resolution of the potential measurement. A sample containing SMPs in sucrose buffer, supplemented with Oxonol VI ( $2 \mu \mathrm{M}$ after mixing) was mixed 1:1 with a sucrose buffer containing ATP ( $1 \mathrm{mM}$ after mixing). Changes in absorbance were monitored at $623 \mathrm{~nm}$ (relative to those measured at $604 \mathrm{~nm}$ ). The SMP concentration was $0.1 \mathrm{mg} \mathrm{ml}^{-1}$ after mixing.

The oxonol VI response to the membrane potential was calibrated using asolectin liposomes of the same size as the SMPs. The liposomes were prepared from washed soybean lipids (L-a-phosphatidylcholine, type II-S) essentially as described in $^{60}$. The preparation buffer contained $20 \mathrm{mM}$ Hepes $\mathrm{pH} 8,150 \mathrm{mM}$ $\mathrm{NaCl}$ and $0.5 \mathrm{mM} \mathrm{KCl}$. A solution of asolectin (total $60 \mathrm{mg}$ ) was prepared at 40 $\mathrm{mg} \mathrm{ml}^{-1}$ and $2 \%$ sodium cholate was added. The lipids were sonicated for 4 min in $15 \mathrm{~s}$ on and $15 \mathrm{~s}$ off cycles using a Vibra cell sonicator (Sonics \& Materials Inc.) with the amplitude set to $35 \%$ of the maximal. After formation of the liposomes detergent was removed by three-step dialysis in the same buffer, but without detergent $(200 \mathrm{ml}$ for $3 \mathrm{~h}, 300 \mathrm{ml}$ for $3 \mathrm{~h}$ and $500 \mathrm{ml}$ overnight).

The calibration was performed by gradually increasing the external $\mathrm{KCl}$ concentration in the presence of valinomycin and monitoring the associated absorbance changes of the dye at $623 \mathrm{~nm}$. The membrane potential associated with the dye response to the potassium gradient was calculated using the Nernst equation. The lipid concentration was $8 \mathrm{mg} \mathrm{ml}^{-1}, 2 \mu \mathrm{M}$ oxonol VI and $1 \mu \mathrm{M}$ valinomycin was used. External potassium concentrations were (in units of $\mathrm{mM}): 2.5,5,10,20,40$ and $50(0.5 \mathrm{mM} \mathrm{KCl}$ inside). The buffer composition was the same as that used for liposome preparation and the final volume was $1 \mathrm{ml}$.

To measure changes in proton concentration, the SMPs were dissolved in a sucrose buffer to $0.1 \mathrm{mg} \mathrm{ml}^{-1}$ and the fluorescent dye ACMA ${ }^{61}$ was added (200 $\mathrm{nM})$. The sample was excited at $410 \mathrm{~nm}$ and emission was recorded at $480 \mathrm{~nm}$. A baseline was recorded for $\sim 60 \mathrm{~s}$ and then proton pumping was initiated by addition of ATP $(160 \mu \mathrm{M}$ final concentration). Measurements were performed using a fluorometer (Cary Eclipse fluorescence spectrophotometer, model G9800 A, Agilent)

Respiratory control ratio. The RCR was determined by measuring the oxygenreduction rate, using a Clark-type electrode (Hansatech Instruments), upon addition of the SMPs, in the absence and presence of the proton ionophore FCCP. All measurements were performed in the buffer used to dissolve the SMPs (see Preparation of SMPs above), with the addition of fatty-acid free BSA $\left(1 \mathrm{mg} \mathrm{ml}^{-1}\right)$. Succinate was added to the measurement chamber (final concentration $10 \mathrm{mM}$ ) and a background was recorded. SMPs, pre-incubated with $10 \mathrm{mM}$ succinate on ice for $15 \mathrm{~min}$ to activate complex II, were then added (final concentration $0.09 \mathrm{mg} \mathrm{ml}$

${ }^{-1}$ ) and the oxygen reduction was monitored before and after addition of FCCP at $1 \mu \mathrm{M}$ final concentration.

Data availability. Data supporting the findings of this manuscript are available from the corresponding author upon reasonable request.

Received: 15 January 2018 Accepted: 9 July 2018

Published online: 09 August 2018

\section{References}

1. Ferguson-Miller, S., Hiser, C. \& Liu, J. Gating and regulation of the cytochrome $c$ oxidase proton pump. Biochim. Biophys. Acta 1817, 489-494 (2012).

2. Rich, P. R. \& Maréchal, A. Functions of the hydrophilic channels in protonmotive cytochrome $c$ oxidase. J. R. Soc. Interface 10, 183-196 (2013).

3. Blomberg, M. R. A. \& Siegbahn, P. E. M. Proton pumping in cytochrome $c$ oxidase: energetic requirements and the role of two proton channels. Biochim. Biophys. Acta. 1837, 1165-1177 (2014).

4. Popović, D. M., Leontyev, I. V., Beech, D. G. \& Stuchebrukhov, A. A. Similarity of cytochrome $c$ oxidases in different organisms. Protein.: Struct., Funct. Bioinforma. 78, 2691-2698 (2010).
5. Von Ballmoos, C., Ädelroth, P., Gennis, R. B. \& Brzezinski, P. Proton transfer in $b a_{3}$ cytochrome $c$ oxidase from Thermus thermophilus. Biochim. Biophys. Acta 1817, 650-657 (2012).

6. Wikström, M., Sharma, V., Kaila, V. R. I., Hosler, J. P. \& Hummer, G. New perspectives on proton pumping in cellular respiration. Chem. Rev. 115, 2196-2221 (2015)

7. Siletsky, S., Kaulen, A. D. \& Konstantinov, A. A. Resolution of electrogenic steps coupled to conversion of cytochrome $c$ oxidase from the peroxy to the ferryl-oxo state. Biochemistry 38, 4853-4861 (1999).

8. Jasaitis, A., Verkhovsky, M. I., Morgan, J. E., Verkhovskaya, M. L. \& Wikström, M. Assignment and charge translocation stoichiometries of the major electrogenic phases in the reaction of cytochrome $c$ oxidase with dioxygen. Biochemistry 38, 2697-2706 (1999).

9. Ruitenberg, M., Kannt, A., Bamberg, E., Fendler, K. \& Michel, H. Reduction of cytochrome $c$ oxidase by a second electron leads to proton translocation. Nature 417, 99-102 (2002).

10. Mills, D. A. \& Ferguson-Miller, S. Influence of structure, $\mathrm{pH}$ and membrane potential on proton movement in cytochrome oxidase. Biochim. Biophys. Acta 1555, 96-100 (2002).

11. Gregory, L. \& Ferguson-Miller, S. Independent control of respiration in cytochrome $c$ oxidase vesicles by $\mathrm{pH}$ and electrical gradients. Biochemistry 28, 2655-2662 (1989).

12. Hosler, J. P., Ferguson-Miller, S. \& Mills, D. A. Energy transduction: proton transfer through the respiratory complexes. Annu. Rev. Biochem. 75, 165-187 (2006).

13. Grivennikova, V. G., Gavrikova, E. V. \& Vinogradov, A. D. An increase of the energy coupling capacity of submitochondrial particles by lanthanides. FEBS Lett. 347, 243-246 (1994).

14. Lee, C. P., Ernster, L. \& Chance, B. Studies of the energy-transfer system of submitochondrial particles: kinetic studies of the effect of oligomycin on the respiratory chain of EDTA particles. Eur. J. Biochem. 8, 153-163 (1969).

15. Poiana, F. et al. Splitting of the O-O bond at the heme-copper catalytic site of respiratory oxidases. Science Advances 3, 6 (2017).

16. Proshlyakov, D. A. et al. Oxygen activation and reduction in respiration: involvement of redox-active tyrosine 244. Science 290, 1588-1591 (2000).

17. Kitagawa, T. \& Ogura, T. Time-resolved resonance Raman investigation of oxygen reduction mechanism of bovine cytochrome $c$ oxidase. J. Bioenerg. Biomembr. 30, 71-79 (1998).

18. Karpefors, M., Ädelroth, P., Namslauer, A., Zhen, Y. J. \& Brzezinski, P. Formation of the "peroxy" intermediate in cytochrome $c$ oxidase is associated with internal proton/hydrogen transfer. Biochemistry 39, 14664-14669 (2000).

19. Hill, B. C. \& Greenwood, C. The reaction of fully reduced cytochrome $c$ oxidase with oxygen studied by flow-flash spectrophotometry at room temperature. Biochem J. 218, 913-921 (1984).

20. Ädelroth, P., Ek, M. \& Brzezinski, P. Factors determining electron-transfer rates in cytochrome $c$ oxidase: investigation of the oxygen reaction in the $R$. sphaeroides and bovine enzymes. Biochim. Biophys. Acta 1367, 107-117 (1998).

21. Sucheta, A., Szundi, I. \& Einarsdottir, O. Intermediates in the reaction of fully reduced cytochrome $c$ oxidase with dioxygen. Biochemistry 37, 17905-17914 (1998).

22. Namslauer, A., Aagaard, A., Katsonouri, A. \& Brzezinski, P. Intramolecular proton-transfer reactions in a membrane-bound proton pump: the effect of $\mathrm{pH}$ on the peroxy to ferryl transition in cytochrome $c$ oxidase. Biochemistry 42, 1488-1498 (2003).

23. Belevich, I. \& Verkhovsky, M. I. Molecular mechanism of proton translocation by cytochrome $c$ oxidase. Antioxid. Redox Signal 10, 1-29 (2008).

24. Blomberg, M. R. A. Mechanism of oxygen reduction in cytochrome $c$ oxidase and the role of the active site tyrosine. Biochemistry 55, 489-500 (2016).

25. Sharma, V. \& Wikström, M. The role of the K-channel and the active-site tyrosine in the catalytic mechanism of cytochrome $c$ oxidase. Biochim. Biophys. Acta. 1857, 1111-1115 (2016).

26. Brändén, G., Gennis, R. B. \& Brzezinski, P. Transmembrane proton translocation by cytochrome $c$ oxidase. Biochim Biophys. Acta 1757, 1052-1063 (2006)

27. Zaslavsky, D., Smirnova, I. A., Ädelroth, P., Brzezinski, P. \& Gennis, R. B. Observation of a novel transient ferryl complex with reduced $\mathrm{Cu}_{\mathrm{B}}$ in cytochrome $c$ oxidase. Biochemistry 38, 2307-2311 (1999).

28. Zaslavsky, D., Kaulen, A. D., Smirnova, I. A., Vygodina, T. \& Konstantinov, A A. Flash-induced membrane potential generation by cytochrome $c$ oxidase. FEBS Lett. 336, 389-393 (1993).

29. Mitchell, P. \& Moyle, J. Evidence discriminating between the chemical and the chemiosmotic mechanisms of electron transport phosphorylation. Nature 208, 1205-1206 (1965)

30. Racker, E. \& Horstman, L. L. Partial resolution of the enzymes catalyzing oxidative phosphorylation. 13. Structure and function of submitochondrial particles completely resolved with respect to coupling factor. J. Biol. Chem. 242, 2547-2551 (1967). 
31. Bashford, C. L. \& Thayer, W. S. Thermodynamics of the electrochemical proton gradient in bovine heart submitochondrial particles. J. Biol. Chem. 252, 8459-8463 (1977).

32. Sjöholm, J. et al. The lateral distance between a proton pump and ATP synthase determines the ATP-synthesis rate. Sci. Rep. 7, 1 (2017).

33. Vanneste, W. H. The stoichiometry and absorption spectra of components $a$ and $a_{3}$ in cytochrome $c$ oxidase. Biochemistry 5, 838-848 (1966).

34. Liao, G. L. \& Palmer, G. The reduced minus oxidized difference spectra of cytochromes $a$ and $a_{3}$. Biochim. Biophys. Acta 1274, 109-111 (1996).

35. Karpefors, M. et al. Electron-proton interactions in terminal oxidases. Biochim Biophys. Acta 1365, 159-169 (1998).

36. Faxén, K., Gilderson, G., Ädelroth, P. \& Brzezinski, P. A mechanistic principle for proton pumping by cytochrome $c$ oxidase. Nature 437, 286-289 (2005).

37. Verkhovsky, M. I., Morgan, J. E., Verkhovskaya, M. L. \& Wikström, M. Translocation of electrical charge during a single turnover of cytochrome-c oxidase. Biochim. Biophys. Acta 1318, 6-10 (1997).

38. Salomonsson, L., Faxén, K., Ädelroth, P. \& Brzezinski, P. The timing of proton migration in membrane-reconstituted cytochrome $c$ oxidase. Proc. Natl Acad. Sci. USA 102, 17624-17629 (2005).

39. Karpefors, M., Ädelroth, P., Aagaard, A., Smirnova, I. A. \& Brzezinski, P. The deuterium isotope effect as a tool to investigate enzyme catalysis: protontransfer control mechanisms in cytochrome $c$ oxidase. Isr. J. Chem. 39, 427-437 (1999).

40. Belevich, I., Bloch, D. A., Belevich, N., Wikström, M. \& Verkhovsky, M. I. Exploring the proton pump mechanism of cytochrome $c$ oxidase in real time. Proc. Natl Acad. Sci. USA 104, 2685-2690 (2007).

41. Brzezinski, P. \& Larsson, G. Redox-driven proton pumping by heme-copper oxidases. Biochim. Biophys. Acta 1605, 1-13 (2003).

42. Wikström, M. \& Morgan, J. E. The dioxygen cycle-spectral, kinetic, and thermodynamic characteristics of ferryl and peroxy intermediates observed by reversal of the cytochrome-oxidase reaction. J. Biol. Chem. 267, 10266-10273 (1992).

43. Belevich, I., Verkhovsky, M. I. \& Wikström, M. Proton-coupled electron transfer drives the proton pump of cytochrome $c$ oxidase. Nature 440, 829-832 (2006).

44. Karpefors, M., Ädelroth, P., Zhen, Y., Ferguson-Miller, S. \& Brzezinski, P. Proton uptake controls electron transfer in cytochrome $c$ oxidase. Proc. Natl Acad. Sci. USA 95, 13606-13611 (1998).

45. Salomonsson, L., Brändén, G. \& Brzezinski, P. Deuterium isotope effect of proton pumping in cytochrome $c$ oxidase. Biochim. Biophys. Acta 1777, 343-350 (2008).

46. Popović, D. M. \& Stuchebrukhov, A. A. Proton pumping mechanism and catalytic cycle of cytochrome $c$ oxidase: Coulomb pump model with kinetic gating. FEBS Lett. 566, 126-130 (2004).

47. Blomberg, M. R. A. \& Siegbahn, P. E. M. The mechanism for proton pumping in cytochrome $c$ oxidase from an electrostatic and quantum chemical perspective. Biochim. Biophys. Acta. 1817, 495-505 (2012).

48. Arnold, S. The power of life-cytochrome $c$ oxidase takes center stage in metabolic control, cell signalling and survival. Mitochondrion 12, 46-56 (2012).

49. Hayashi, T. et al. Higdla is a positive regulator of cytochrome $c$ oxidase. Proc. Natl Acad. Sci. USA 112, 1553-1558 (2015).

50. Schäfer, J., Dawitz, H., Ott, M., Ädelroth, P. \& Brzezinski, P. Structural and functional heterogeneity of cytochrome $c$ oxidase in S. cerevisiae. Biochim. Biophys. Acta https://doi.org/10.1016/j.bbabio.2018.05.004 (2018).

51. Garlich, J., Strecker, V., Wittig, I. \& Stuart, R. A. Mutational analysis of the QRRQ motif in the yeast higl type 2 protein Rcfl reveals a regulatory role for the cytochrome $c$ oxidase complex. J. Biol. Chem. 292, 5216-5226 (2017).

52. Iwata, S., Ostermeier, C., Ludwig, B. \& Michel, H. Structure at 2.8 Å resolution of cytochrome $c$ oxidase from Paracoccus denitrificans. Nature 376, 660-669 (1995).

53. Tsukihara, T. et al. The whole structure of the 13-subunit oxidized cytochrome $c$ oxidase at $2.8 \AA$ A Science 272, 1136-1144 (1996).
54. Svensson-Ek, M. et al. The X-ray crystal structures of wild-type and EQ(I-286) mutant cytochrome $c$ oxidases from Rhodobacter sphaeroides. J. Mol. Biol. 321, 329-339 (2002).

55. Qin, L., Hiser, C., Mulichak, A., Garavito, R. M. \& Ferguson-Miller, S. Identification of conserved lipid/detergent-binding sites in a high-resolution structure of the membrane protein cytochrome $c$ oxidase. Proc. Natl Acad. Sci. USA 103, 16117-16122 (2006).

56. Smith, A. L. Preparation, properties, and conditions for assay of mitochondria: slaughterhouse material, small-scale. Meth. Enzymol. 10, 81-86 (1967).

57. Brändén, M. et al. On the role of the K-proton transfer pathway in cytochrome c oxidase. Proc. Natl Acad. Sci. USA 98, 5013-5018 (2001).

58. Rydström Lundin, C., Von Ballmoos, C., Ott, M., Ädelroth, P. \& Brzezinski, $\mathrm{P}$. Regulatory role of the respiratory supercomplex factors in Saccharomyces cerevisiae. Proc. Natl Acad. Sci. USA 113, E4476-E4485 (2016).

59. Smith, J. C. Potential-sensitive molecular probes in membranes of bioenergetic relevance. Biochim. Biophys Acta. 1016, 1-28 (1990).

60. Kagawa, Y. \& Racker, E. Partial resolution of the enzymes catalyzing oxidative phosphorylation. XXV. Reconstitution of vesicles catalyzing inorganic phosphorus-32-adenosine triphosphate exchange. J. Biol. Chem. 246, 5477-5487 (1971).

61. Casadio, R. Measurements of transmembrane $\mathrm{pH}$ differences of low extents in bacterial chromatophores-a study with the fluorescent probe 9-amino, 6chloro, 2-methoxyacridine. Eur. Biophys. J. 19, 189-201 (2004).

\section{Acknowledgements}

We would like to acknowledge Drs. Pia Ädelroth, Christoph von Ballmoos and Irina Smirnova for valuable advice and discussions. We would also like to thank Jacob Schäfer for help in preparation of Fig. 1. These studies were supported by grants from the Knut and Alice Wallenberg Foundation (KAW) and the Swedish Research Council (VR).

\section{Author contributions}

P.B. conceived and supervised the research, M.B. prepared samples and performed the experiments, P.B. and M.B. evaluated the data and wrote the manuscript.

\section{Additional information}

Supplementary Information accompanies this paper at https://doi.org/10.1038/s41467018-05615-5.

Competing interests: The authors declare no competing interests.

Reprints and permission information is available online at http://npg.nature.com/ reprintsandpermissions/

Publisher's note: Springer Nature remains neutral with regard to jurisdictional claims in published maps and institutional affiliations.

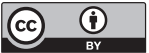

Open Access This article is licensed under a Creative Commons Attribution 4.0 International License, which permits use, sharing, adaptation, distribution and reproduction in any medium or format, as long as you give appropriate credit to the original author(s) and the source, provide a link to the Creative Commons license, and indicate if changes were made. The images or other third party material in this article are included in the article's Creative Commons license, unless indicated otherwise in a credit line to the material. If material is not included in the article's Creative Commons license and your intended use is not permitted by statutory regulation or exceeds the permitted use, you will need to obtain permission directly from the copyright holder. To view a copy of this license, visit http://creativecommons.org/ licenses/by/4.0/.

(c) The Author(s) 2018 